\title{
Nonholonomic Geometry of Viscoanelastic Media and Experimental Confirmation
}

\author{
Armando Ciancio ${ }^{1}$ and Carlo Cattani ${ }^{2}$ \\ ${ }^{1}$ Department of Mathematics and Computer Science, University of Messina, Viale F. Stagno d'Alcontres 31, 98166 Messina, Italy \\ ${ }^{2}$ Department of Mathematics, University of Salerno, Via Ponte Don Melillo, 84084 Fisciano, Italy
}

Correspondence should be addressed to Armando Ciancio; aciancio@unime.it

Received 16 July 2013; Accepted 9 August 2013

Academic Editor: Cristian Toma

Copyright (C) 2013 A. Ciancio and C. Cattani. This is an open access article distributed under the Creative Commons Attribution License, which permits unrestricted use, distribution, and reproduction in any medium, provided the original work is properly cited.

A thermodynamical model for viscoanelastic media is analyzed using the nonholonomic geometry. A 27-dimensional manifold is introduced, and the differential equations for the geodetics are determined and analytically solved. It is shown that, in this manifold, the best specific entropy is a harmonic function. In the linear case the propagation of transverse acoustic waves is studied, and the theoretical results are compared with some experimental data from a polymeric material (polyisobutylene).

\section{Introduction}

From macroscopic point of view the most popular mathematical approaches to nonequilibrium thermodynamics are based both on the Caratheodory theory [1-3] which involves Pfaff equations and on the contact structure of thermodynamic state space [4-6]. If these equations are completely integrable, then the thermodynamics is called holonomic otherwise nonholonomic.

The theory of nonequilibrium holonomic thermodynamics for mechanical phenomena in continuous media was developed in the 90s (see e.g., [7] and references therein). The phenomenological equations were derived $[8,9]$ by introducing the tensorial internal variables $\varepsilon_{\alpha \beta}^{(1)}(\alpha, \beta=$ $1,2,3)$ which occur in the entropy production.

In particular, if one linearizes this theory by neglecting the cross effects among the irreversible phenomena (heat flow, mechanical viscosity, and anelastic deformations), the following rheological equations for distortional phenomena are obtained [10]:

$$
\begin{aligned}
\frac{d \widetilde{\tau}_{\alpha \beta}}{d t}+R_{(d) 0}^{(\tau)} \widetilde{\tau}_{\alpha \beta}= & R_{(d) 2}^{(\varepsilon)} \frac{d^{2} \widetilde{\varepsilon}_{\alpha \beta}}{d t^{2}}+R_{(d) 1}^{(\varepsilon)} \frac{d \widetilde{\varepsilon}_{\alpha \beta}}{d t} \\
& +R_{(d) 0}^{(\varepsilon)} \widetilde{\varepsilon}_{\alpha \beta},
\end{aligned}
$$

where $\widetilde{\tau}_{\alpha \beta}$ and $\widetilde{\varepsilon}_{\alpha \beta}$ are the deviators of the stress $\left(\tau_{\alpha \beta}\right)$ and the strain $\left(\varepsilon_{\alpha \beta}\right)$ tensors, respectively.

The quantities $R$ are given by

$$
\begin{gathered}
R_{(d) 0}^{(\tau)}=a^{(1,1)} \eta_{s}^{(1,1)}=\frac{1}{\sigma} \geq 0, \\
R_{(d) 0}^{(\varepsilon)}=a^{(0,0)}\left(a^{(1,1)}-a^{(0,0)}\right) \eta_{s}^{(1,1)} \geq 0, \\
R_{(d) 1}^{(\varepsilon)}=a^{(0,0)}+a^{(1,1)} \eta_{s}^{(1,1)} \eta_{s}^{(0,0)} \geq 0, \\
R_{(d) 2}^{(\varepsilon)}=\eta_{s}^{(0,0)} \geq 0,
\end{gathered}
$$

in which $\sigma$ is the relaxation time and $a^{(1,1)} \geq a^{(0,0)} \geq 0$ are the state coefficients, while $\eta_{s}^{(0,0)}$ and $\eta_{s}^{(1,1)}$ are the phenomenological coefficients related to the following physical phenomena:

$$
\begin{gathered}
a^{(0,0)} \Longrightarrow \text { elasticity, } \\
a^{(1,1)} \Longrightarrow \text { anelasticity, } \\
\eta_{s}^{(0,0)} \Longrightarrow \text { viscosity, } \\
\eta_{s}^{(1,1)} \Longrightarrow \text { fluidity. }
\end{gathered}
$$

In this paper we reconsider the theory from the point of view of the nonholonomic geometry. 
In Sections 2 and 3 the analytic properties of the entropy are discussed, and in Section 4 the differential equations of geodetics in the space of state are obtanied.

Finally, in Section 5 we study the transverse waves and we will show the connection between complex numbers and the shear complex modulus. By applying these results to a polymeric material, as polyisobutylene we will also show that the expected results of the theoretical model are in agreement with the experimental data.

\section{Gibbs-Pfaff Equation of Viscoanelastic Media}

Let us define the space of states

$$
R^{27}=R^{2 \cdot 13+1}=\left\{s, T, u, \tau_{\alpha \beta}^{(\mathrm{eq})}, \varepsilon_{\alpha \beta}, \tau_{\alpha \beta}^{(1)}, \varepsilon_{\alpha \beta}^{(1)}\right\},
$$

where $s$ is the specific entropy, $T$ is the absolute temperature, $u$ is the specific internal energy, $\tau_{\alpha \beta}^{(\mathrm{eq})}$ is the symmetric equilibrium stress tensor, $\varepsilon_{\alpha \beta}$ is the total symmetric strain tensor, and $\tau_{\alpha \beta}^{(1)}$ is the symmetric affinity stress tensor conjugate to the anelastic tensor $\varepsilon_{\alpha \beta}^{(1)}$ (the symmetric tensorial thermodynamic internal variable).

Let $v$ be the specific volume related to the mass density $\varrho=$ constant (homogeneous media) by $v \varrho=1$. The GibbsPfaff equation of viscoanelastic media is

$$
T d s=d u-v \tau_{\alpha \beta}^{(\mathrm{eq})} d \varepsilon_{\alpha \beta}+v \tau_{\alpha \beta}^{(1)} d \varepsilon_{\alpha \beta}^{(1)}
$$

This equation defines in $R^{27}$ the nonholonomic contact distribution of dimension 26 so that the highest dimension of integral manifold is 13 .

The $C^{\infty}$ representation of this integral manifold (of maximum dimension) is usually given as follows:

$$
\begin{gathered}
s=\Phi\left(u, \varepsilon_{\alpha \beta}, \varepsilon_{\alpha \beta}^{(1)}\right), \\
T^{-1}=\frac{\partial \Phi}{\partial u}\left(u, \varepsilon_{\alpha \beta}, \varepsilon_{\alpha \beta}^{(1)}\right), \\
\tau_{\alpha \beta}^{(\mathrm{eq})}=-\varrho T \frac{\partial \Phi}{\partial \varepsilon_{\alpha \beta}}\left(u, \varepsilon_{\alpha \beta}, \varepsilon_{\alpha \beta}^{(1)}\right), \\
\tau_{\alpha \beta}^{(1)}=\varrho T \frac{\partial \Phi}{\partial \varepsilon_{\alpha \beta}^{(1)}}\left(u, \varepsilon_{\alpha \beta}, \varepsilon_{\alpha \beta}^{(1)}\right) .
\end{gathered}
$$

This parametrization is based on the arbitrary $C^{\infty}$-function $\Phi$. So that we have a family of integral manifolds of dimension 13 indexed on the arbitrary function $\Phi$.

The state parameters

$$
T^{-1}, T^{-1} \varrho^{-1} \tau_{\alpha \beta}^{(\mathrm{eq})}, T^{-1} \varrho^{-1} \tau_{\alpha \beta}^{(1)}
$$

are related by the equations of motion and the equations of state $[8,9]$.

The $C^{\infty}$-representation is a generic element in the 1 -jet space converted into

$$
\left(u, \varepsilon_{\alpha \beta}, \varepsilon_{\alpha \beta}^{(1)}, s\left(u, \varepsilon_{\alpha \beta}, \varepsilon_{\alpha \beta}^{(1)}\right), \frac{\partial s}{\partial u},-\frac{\partial s}{\partial \varepsilon_{\alpha \beta}}, \frac{\partial s}{\partial \varepsilon_{\alpha \beta}^{(1)}}\right) .
$$

In order to fix a representative for the specific entropy $s=$ $\Phi\left(u, \varepsilon_{\alpha \beta}, \varepsilon_{\alpha \beta}^{(1)}\right)$, we need a supplementary condition as follows.

Theorem 1. If $s=\Phi\left(u, \varepsilon_{\alpha \beta}, \varepsilon_{\alpha \beta}^{(1)}\right)$ is an homogeneous function of order one, then

$$
\Phi=\frac{u}{T}-\frac{1}{\varrho T} \tau_{\alpha \beta}^{(\mathrm{eq})} \varepsilon_{\alpha \beta}+\frac{1}{\varrho T} \tau_{\alpha \beta}^{(1)} \varepsilon_{\alpha \beta}^{(1)}
$$

Proof. The condition of homogeneity of order one

$$
\Phi\left(k u, k \varepsilon_{\alpha \beta}, k \varepsilon_{\alpha \beta}^{(1)}\right)=k \Phi\left(u, \varepsilon_{\alpha \beta}, \varepsilon_{\alpha \beta}^{(1)}\right)
$$

gives the PDE

$$
u \frac{\partial \Phi}{\partial u}+\varepsilon_{\alpha \beta} \frac{\partial \Phi}{\partial \varepsilon_{\alpha \beta}}+\varepsilon_{\alpha \beta}^{(1)} \frac{\partial \Phi}{\partial \varepsilon_{\alpha \beta}^{(1)}}=\Phi\left(u, \varepsilon_{\alpha \beta}, \varepsilon_{\alpha \beta}^{(1)}\right) .
$$

Then the entropy (solution of this PDE) appears as the potential (9).

Corollary 2. If the specific entropy $s=\Phi\left(u, \varepsilon_{\alpha \beta}, \varepsilon_{\alpha \beta}^{(1)}\right)$ is an homogeneous function of order one, then

(1) the variables $s, u, \varepsilon_{\alpha \beta}, \varepsilon_{\alpha \beta}^{(1)}$ are conjugated to the intensive variables $T, \tau_{\alpha \beta}^{(e q)}, \tau_{\alpha \beta}^{(1)}$;

(2) the variables $s, u, \varepsilon_{\alpha \beta}, \varepsilon_{\alpha \beta}^{(1)}$ are not essential parameters (because they are not independent).

Proof. From the expression (9) we find

$$
\begin{aligned}
T d s+s d T= & d u-\frac{1}{\varrho}\left(\varepsilon_{\alpha \beta} d \tau_{\alpha \beta}^{(\mathrm{eq})}+\tau_{\alpha \beta}^{(\mathrm{eq})} d \varepsilon_{\alpha \beta}\right) \\
& +\frac{1}{\varrho}\left(\varepsilon_{\alpha \beta}^{(1)} d \tau_{\alpha \beta}^{(1)}+\tau_{\alpha \beta}^{(1)} d \varepsilon_{\alpha \beta}^{(1)}\right) .
\end{aligned}
$$

(1) Replacing the relation (5), we get

$$
s d T=-\frac{1}{\varrho} \varepsilon_{\alpha \beta} d \tau_{\alpha \beta}^{(\mathrm{eq})}+\frac{1}{\varrho} \varepsilon_{\alpha \beta}^{(1)} d \tau_{\alpha \beta}^{(1)},
$$

and the first statement is true.

(2) The foregoing relation shows that, for example,

$$
\frac{\varepsilon_{\alpha \beta}}{s}, \quad \frac{\varepsilon_{\alpha \beta}^{(1)}}{s}
$$

are essential parameters.

\section{Specific Entropy via Least Squares Lagrangian}

The most convenient way to fix a representative $\Phi$ of the specific entropy $s$ is to look at (5) as a partial derivative 
evolution equation and to build the least squares method Lagrangian $L$

$$
\begin{aligned}
2 L= & \left\|T^{-1}-\frac{\partial \Phi}{\partial u}\right\|^{2}+\left\|\tau_{\alpha \beta}^{(\mathrm{eq})}+\rho T \frac{\partial \Phi}{\partial \varepsilon_{\alpha \beta}}\right\|^{2} \\
& +\left\|\tau_{\alpha \beta}^{(1)}-\rho T \frac{\partial \Phi}{\partial \varepsilon_{\alpha \beta}^{(1)}}\right\|
\end{aligned}
$$

and the functional

$$
\int_{\Omega} L\left(u, \varepsilon_{\alpha \beta}, \varepsilon_{\alpha \beta}^{(1)}, \Phi, \Phi_{u}, \Phi_{\varepsilon_{\alpha \beta}}, \Phi_{\varepsilon_{\alpha \beta}^{(1)}}\right) d \Omega,
$$

where $\Phi_{u}=\partial \Phi / \partial u, \Phi_{\varepsilon_{\alpha \beta}}=\partial \Phi / \partial \varepsilon_{\alpha \beta}$, and $\Phi_{\varepsilon_{\alpha \beta}^{(1)}}=\partial \Phi / \partial \varepsilon_{\alpha \beta}^{(1)}$.

The extremals are solutions of the Euler-Lagrange PDE

$$
\frac{\partial L}{\partial \Phi}-\left(D_{u} \frac{\partial L}{\partial \Phi_{u}}+D_{\varepsilon_{\alpha \beta}} \frac{\partial L}{\partial \Phi_{\varepsilon_{\alpha \beta}}}+D_{\varepsilon_{\alpha \beta}^{(1)}} \frac{\partial L}{\partial \Phi_{\varepsilon_{\alpha \beta}^{(1)}}}\right)=0,
$$

where $D_{*}$ is the total derivative with respect to the variable $*$.

In our case, we get

$$
\begin{gathered}
\frac{\partial L}{\partial \Phi}=0, \quad \frac{\partial L}{\partial \Phi_{u}}=-\left(T^{-1}-\frac{\partial \Phi}{\partial u}\right), \\
\frac{\partial L}{\partial \Phi_{\varepsilon_{\alpha \beta}}}=\rho T\left(\tau_{\alpha \beta}^{(\mathrm{eq})}+\rho T \frac{\partial \Phi}{\partial \Phi_{\varepsilon_{\alpha \beta}}}\right), \\
\frac{\partial L}{\partial \Phi_{\varepsilon_{\alpha \beta}^{(1)}}}=-\rho T\left(\tau_{\alpha \beta}^{(1)}-\rho T \frac{\partial \Phi}{\partial \Phi_{\varepsilon_{\alpha \beta}^{(1)}}}\right) .
\end{gathered}
$$

So that, by replacing the partial derivatives of $L$ in (17), there follows the Laplace equation for the entropy

$$
\frac{\partial^{2} \Phi}{\partial u^{2}}+\rho^{2} T^{2} \frac{\partial^{2} \Phi}{\partial \varepsilon_{\alpha \beta}^{2}}+\rho^{2} T^{2} \frac{\partial^{2} \Phi}{\partial \varepsilon_{\alpha \beta}^{(1)^{2}}}=0 .
$$

Consequently, we have the following.

Theorem 3. The best entropy for the nonholonomic nonequilibrium thermodynamics is an harmonic function.

\section{Geodesics}

Any curve in the distribution (5) is described by

$$
T(t) \dot{s}(t)=\dot{u}(t)-v \tau_{\alpha \beta}^{(\mathrm{eq})}(t) \dot{\varepsilon}_{\alpha \beta}(t)+v \tau_{\alpha \beta}^{(1)}(t) \dot{\varepsilon}_{\alpha \beta}^{(1)}(t) .
$$

In order to be a geodesic, this curve must minimize the energy functional

$$
\begin{aligned}
J=\frac{1}{2} \int_{t_{0}}^{t_{1}}\left(\dot{T}^{2}(t)+\dot{s}^{2}(t)+\dot{u}^{2}(t)+\delta_{\alpha \gamma} \delta_{\beta \delta} \dot{\tau}_{\alpha \beta}^{(\mathrm{eq})} \dot{\tau}_{\gamma \delta}^{(\mathrm{eq})}\right. \\
+\delta_{\alpha \gamma} \delta_{\beta \delta} \dot{\tau}_{\alpha \beta}^{(1)} \dot{\tau}_{\gamma \delta}^{(1)}+\delta_{\alpha \gamma} \delta_{\beta \delta} \dot{\varepsilon}_{\alpha \beta} \dot{\varepsilon}_{\gamma \delta} \\
\left.+\delta_{\alpha \gamma} \delta_{\beta \delta} \dot{\varepsilon}_{\alpha \beta}^{(1)} \dot{\varepsilon}_{\gamma \delta}^{(1)}\right) d t
\end{aligned}
$$

In short, we must solve the problem

$$
\text { min } J \text { subject to }(20) \text {. }
$$

To solve this problem, we use the method of Lagrange multipliers. For this we defined the constrained Lagrangian

$$
\begin{aligned}
L_{1}=\frac{1}{2}( & \dot{T}^{2}(t)+\dot{s}^{2}(t)+\dot{u}^{2}(t)+\delta_{\alpha \gamma} \delta_{\beta \delta} \dot{\tau}_{\alpha \beta}^{(\mathrm{eq})}(t) \dot{\tau}_{\gamma \delta}^{(\mathrm{eq})}(t) \\
& +\delta_{\alpha \gamma} \delta_{\beta \delta} \dot{\tau}_{\alpha \beta}^{(1)}(t) \dot{\tau}_{\gamma \delta}^{(1)}(t) \\
& +\delta_{\alpha \gamma} \delta_{\beta \delta} \dot{\varepsilon}_{\alpha \beta}(t) \dot{\varepsilon}_{\gamma \delta}(t) \\
& \left.+\delta_{\alpha \gamma} \delta_{\beta \delta} \dot{\varepsilon}_{\alpha \beta}^{(1)}(t) \dot{\varepsilon}_{\gamma \delta}^{(1)}(t)\right) \\
+ & \left(T(t) \dot{s}(t)-\dot{u}(t)+v \tau_{\alpha \beta}^{(\mathrm{eq})}(t) \dot{\varepsilon}_{\alpha \beta}(t)\right. \\
& \left.\quad-v \tau_{\alpha \beta}^{(1)}(t) \dot{\varepsilon}_{\alpha \beta}^{(1)}(t)\right)
\end{aligned}
$$

where $p$ is the Lagrangian multiplier.

Theorem 4. The geodesics of the nonholonomic viscoanelastic distribution are solutions of the Euler-Lagrange ODEs for the Lagrangian (23)

$$
\frac{\partial L_{1}}{\partial x^{i}}-D_{t} \frac{\partial L_{1}}{\partial \dot{x}^{i}}=0
$$

where $x^{i}$ are the generalized coordinates:

$$
\begin{array}{rlrl}
x^{1}=T, & x^{2}=s, & x^{3}=u, \\
x_{\alpha \beta}^{4}=\tau_{\alpha \beta}^{(\mathrm{eq})}, & x_{\alpha \beta}^{5}=\varepsilon_{\alpha \beta}, & x_{\alpha \beta}^{6}=\tau_{\alpha \beta}^{(1)}, \\
x_{\alpha \beta}^{7}=\varepsilon_{\alpha \beta}^{(1)} . &
\end{array}
$$

So that explicitly from (23) and (24) we have

$$
\begin{aligned}
& p \dot{s}-\ddot{T}=0, \quad \frac{d}{d t}(\dot{s}+p T)=0, \quad \frac{d}{d t}(\dot{u}-p)=0, \\
& p \nu \dot{\varepsilon}_{\alpha \beta}-\frac{d}{d t} \dot{\tau}_{\alpha \beta}^{(\mathrm{eq})}=0, \quad p \nu \dot{\varepsilon}_{\alpha \beta}^{(1)}+\frac{d}{d t} \dot{\tau}_{\alpha \beta}^{(1)}=0, \\
& \frac{d}{d t}\left(\dot{\varepsilon}_{\alpha \beta}^{(1)}-p v \tau_{\alpha \beta}^{(1)}\right)=0, \quad \frac{d}{d t}\left(\dot{\varepsilon}_{\alpha \beta}+p v \tau_{\alpha \beta}^{(\mathrm{eq})}\right)=0 .
\end{aligned}
$$

The equations (26) with the condition (20) are the differential equations of geodesics.

Let

$$
\begin{aligned}
& T_{0}=T(0), \quad \dot{T}_{0}=\dot{T}(0), \quad s_{0}=s(0), \\
& \dot{s}_{0}=\dot{s}(0), \quad u_{0}=u(0), \quad \dot{u}_{0}=\dot{u}(0) \text {, } \\
& { }_{0}^{(\mathrm{eq})}=\tau_{\alpha \beta}^{(\mathrm{eq})}(0), \quad \dot{\tau}_{\alpha \beta}^{(\mathrm{eq})}=\dot{\tau}_{\alpha \beta}^{(\mathrm{eq})}(0), \\
& \tau_{0 \beta}^{(1)}=\tau_{\alpha \beta}^{(1)}(0), \quad \dot{\tau}_{\alpha \beta}^{(1)}=\dot{\tau}_{\alpha \beta}^{(1)}(0), \\
& \varepsilon_{0} \varepsilon_{\alpha \beta}=\varepsilon_{\alpha \beta}(0), \quad \dot{\varepsilon}_{0 \beta}=\dot{\varepsilon}_{\alpha \beta}(0), \\
& { }_{0}^{(1)}=\varepsilon_{\alpha \beta}^{(1)}(0), \quad \dot{\varepsilon}_{\alpha \beta}^{(1)}=\dot{\varepsilon}_{\alpha \beta}^{(1)}(0)
\end{aligned}
$$

be the given (constant) initial values. 
By some explicit computation we can easily show that the following.

Theorem 5. The geodesics of the nonholonomic viscoanelastic distribution, as solution of the Cauchy problem (26) and (27), are the family of curves:

$$
\begin{aligned}
& u(t)=\dot{u}_{0} t+u_{0} \\
& s(t)=\frac{\dot{T}_{0}}{p} \cos p t+\frac{\dot{s}_{0}}{p} \sin p t+\left(s_{0}-\frac{\dot{T}_{0}}{p}\right) \\
& T(t)=\frac{\dot{T}_{0}}{p} \sin p t-\frac{\dot{s}_{0}}{p} \cos p t+\left(T_{0}+\frac{\dot{s}_{0}}{p}\right)
\end{aligned}
$$

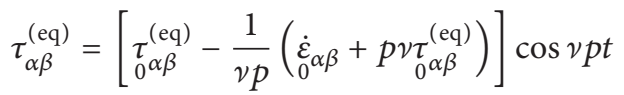

$$
\begin{aligned}
& +\frac{1}{\nu p} \dot{\tau}^{\alpha \beta}(\mathrm{eq}) \sin \nu p t+\frac{1}{\nu p}\left(\dot{\varepsilon}_{0} \dot{\alpha}_{\alpha \beta}+p \nu \tau_{\alpha \beta}^{(\mathrm{eq})}\right)
\end{aligned}
$$

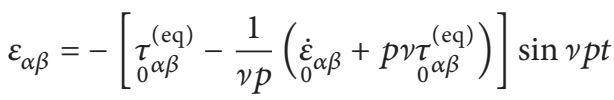

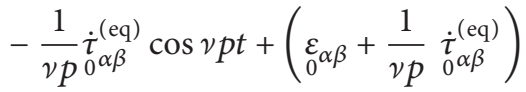

$$
\begin{aligned}
& \tau_{\alpha \beta}^{(1)}=\left[\begin{array}{c}
\tau_{\alpha \beta}^{(1)} \\
0^{\alpha \beta}
\end{array} \frac{1}{\nu p}\left({ }_{0}^{\dot{\varepsilon}_{\alpha \beta}^{(1)}}+\underset{0}{\left.p \nu \tau_{\alpha \beta}^{(1)}\right)}\right)\right] \cos v p t
\end{aligned}
$$

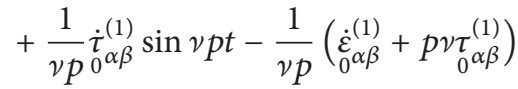

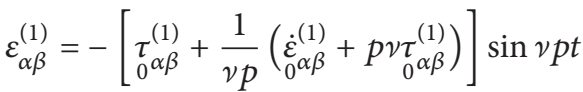

$$
\begin{aligned}
& -\frac{1}{\nu p} \dot{\tau}_{0}^{(1)} \cos \nu p t-\left(\varepsilon_{\alpha \beta}^{(1)}+\frac{1}{\nu p} \dot{\tau}_{0}^{(1)}\right) .
\end{aligned}
$$

Proof. Let us first solve the simplest equation: from $(26)_{3}$ we have

$$
\dot{u}-p=\dot{u}_{0} \Longrightarrow u=\left(\dot{u}_{0}+p\right) t+u_{0}
$$

From $(26)_{1}$ it is

$$
\frac{d}{d t}(p s-\dot{T})=0 \Longrightarrow \dot{T}=p\left(s-s_{0}\right)+\dot{T}_{0}
$$

and deriving $(26)_{2}$

$$
\ddot{s}+p \dot{T}=0 .
$$

By replacing $\dot{T}$ with the previous expression we get

$$
\ddot{s}+p\left[p\left(s-s_{0}\right)+\dot{T}_{0}\right]=0,
$$

that is

$$
\ddot{s}+p^{2} s=\left(p^{2} s_{0}-p \dot{T}_{0}\right) .
$$

This is a linear nonhomogeneous second-order (harmonic) equation whose solution is

$$
s(t)=\left(s_{0}-\frac{\dot{T}_{0}}{p}\right)+\frac{\dot{T}_{0}}{p} \cos p t+\frac{\dot{s}_{0}}{p} \sin p t .
$$

With this function, from the expression

$$
\dot{T}=p\left(s-s_{0}\right)+\dot{T}_{0},
$$

we can compute also $T(t)$ :

$$
\dot{T}=p\left[\left(\left(s_{0}-\frac{\dot{T}_{0}}{p}\right)+\frac{\dot{T}_{0}}{p} \cos p t+\frac{\dot{s}_{0}}{p} \sin p t\right)-s_{0}\right]+\dot{T}_{0} ;
$$

that is,

$$
\dot{T}=\dot{T}_{0} \cos p t+\dot{s}_{0} \sin p t
$$

The solution is

$$
T(t)=\frac{\dot{T}_{0}}{p} \sin p t-\frac{\dot{s}_{0}}{p} \cos p t+\left(T_{0}+\frac{\dot{s}_{0}}{p}\right) .
$$

Concerning $(26)_{4,5,6,7}$ we can notice that it is enough to solve $(26)_{4,7}$ since their solutions are formally equal to the solutions of $(26)_{5,6}$ since these equations coincide with $(26)_{4,7}$ apart from the substitutions:

$$
\varepsilon_{\alpha \beta} \Longrightarrow \varepsilon_{\alpha \beta}^{(1)}, \quad \tau_{\alpha \beta}^{(\mathrm{eq})} \Longrightarrow-\tau_{\alpha \beta}^{(1)} .
$$

Thus from $(26)_{7}$ it is

$$
\dot{\varepsilon}_{\alpha \beta}+p v \tau_{\alpha \beta}^{(\mathrm{eq})}=\dot{\varepsilon}_{\alpha \beta}+p v \tau_{\alpha \beta}^{(\mathrm{eq})}
$$

that is,

$$
\dot{\varepsilon}_{\alpha \beta}=-p \nu \tau_{\alpha \beta}^{(\mathrm{eq})}+\left(\dot{\varepsilon}_{0} \dot{0}_{\alpha \beta}+p \nu \tau_{0 \beta}^{(\mathrm{eq})}\right)
$$

If we put this expression in $(26)_{4}$ we get

$$
p v\left[-p v \tau_{\alpha \beta}^{(\mathrm{eq})}+\left(\dot{\varepsilon}_{0}{ }_{0}+p \underset{0}{p} \tau_{\alpha \beta}^{(\mathrm{eq})}\right)\right]-\frac{d}{d t} \dot{\tau}_{\alpha \beta}^{(\mathrm{eq})}=0,
$$

and by some manipulation we get the (vectorial) linear second order harmonic equation for $\tau_{\alpha \beta}^{(\mathrm{eq})}$ :

$$
\frac{d}{d t} \dot{\tau}_{\alpha \beta}^{(\mathrm{eq})}+p^{2} v^{2} \tau_{\alpha \beta}^{(\mathrm{eq})}=p \nu\left(\dot{\varepsilon}_{0}{ }_{\alpha \beta}+\underset{0}{p \nu \tau_{\alpha \beta}^{(\mathrm{eq})}}\right)
$$

The solution is

$$
\begin{aligned}
& \tau_{\alpha \beta}^{(\mathrm{eq})}=\left[\tau_{0 \beta}^{(\mathrm{eq})}-\frac{1}{\nu p}\left(\dot{\varepsilon}_{0 \beta}+p{ }_{0} \tau_{0}^{(\mathrm{eq})}\right)\right] \cos \nu p t \\
& +\frac{1}{\nu p} \dot{\tau}_{0}^{(\mathrm{eq})} \sin \nu p t+\frac{1}{\nu p}\left(\dot{\varepsilon}_{0 \beta}+\underset{0}{p \nu \tau_{\alpha \beta}^{(\mathrm{eq})}}\right),
\end{aligned}
$$


so that by integrating (41) and using the previous equation, we can easily get the expression for $\varepsilon_{\alpha \beta}$

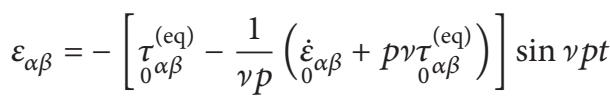

$$
\begin{aligned}
& -\frac{1}{\nu p \dot{\tau}^{\alpha \beta}}{ }^{(\mathrm{eq})} \cos \nu p t+\left(\varepsilon_{0 \beta}+\frac{1}{\nu p \dot{\tau}^{\alpha \beta}} \dot{\mathrm{e}}^{(\mathrm{eq})}\right) .
\end{aligned}
$$

With similar computations we get also the last two equations of (28).

The Lagrangian multiplier $p$ is obtained by inserting the functions (28) and derivatives into (20).

It should be noticed that the the projection of the geodesics (28) into different planes gives rise to well known curves. For instance, in the plane $\langle T, s\rangle(28)$ are the parametric equations of a cycloid. Moreover, by assuming that all the initial values are positive, the asymptotic limits give

$$
\begin{gathered}
\lim _{t \rightarrow \infty} u(t)=+\infty, \\
\lim _{t \rightarrow \infty}|T(t)| \leq\left|T_{0}-\frac{\dot{s}_{0}}{p}\right|+\left|\frac{\dot{T}_{0}}{p}\right|+\left|\frac{\dot{s}_{0}}{p}\right|, \\
\lim _{t \rightarrow \infty}|s(t)| \leq\left|s_{0}-\frac{\dot{T}_{0}}{p}\right|+\left|\frac{\dot{T}_{0}}{p}\right|+\left|\frac{\dot{s}_{0}}{p}\right|,
\end{gathered}
$$

which are in agreement with physical consideration especially for the entropy $s$ which is upper bounded. Analogously we have similar bounded asymptotic limits for the vectorial functions.

\section{Experimental Approach to the Linear Response Theory}

In a previous paper [11] by the application of the linear response theory [12-15] numerical values of (1) were considered, and the results were compared with experimental data.

In this section we study another aspect of the transversal waves propagation in viscoanelastic media, and we apply the theoretical results to a polymeric material as the polyisobutylene.

We consider, with respect to the Cartesian orthogonal axes $\left(x_{1}, x_{2}, x_{3}\right)$, the following displacement $\mathbf{u}$

$$
u_{3}=A e^{i\left(k x_{1}-\omega t\right)}, \quad u_{1}=u_{3}=0
$$

being $i^{2}=-1$ and $k=k_{1}+i k_{2}$ the complex wave number, so that

$$
v_{s}=\frac{\omega}{k_{1}}
$$

is the phase velocity and $k_{2}$ is connected with the attenuation of the waves.

$$
\begin{gathered}
\text { As } \varepsilon_{\alpha \beta}=1 / 2\left(\partial u_{\alpha} / \partial x_{\beta}+\partial u_{\beta} / \partial x_{\alpha}\right), \text { from (1) one obtains } \\
k_{1}=\omega \sqrt{\varrho}\{B(\omega)(\sqrt{1+D(\omega)}+1)\}^{1 / 2}, \\
k_{1}=\omega \sqrt{\varrho}\{B(\omega)(\sqrt{1+D(\omega)}-1)\}^{1 / 2},
\end{gathered}
$$

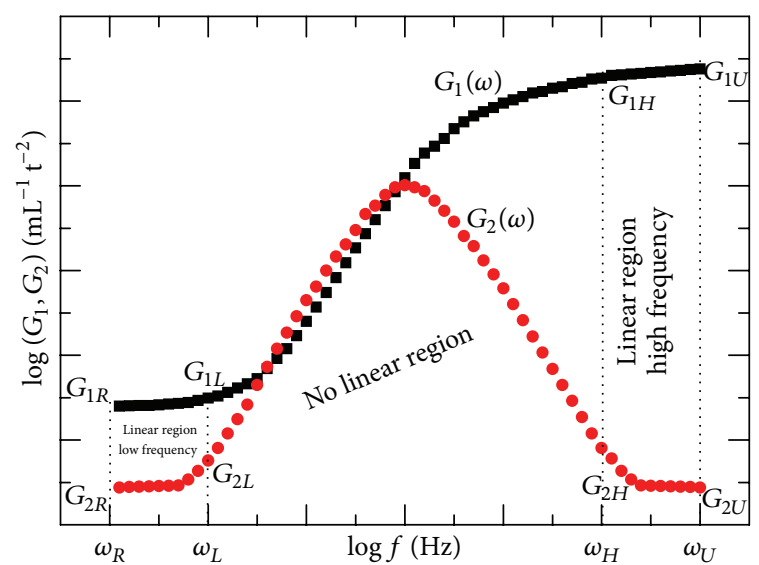

FIGURE 1: Generic storage and loss moduli.

where

$$
\begin{gathered}
B(\omega)=\frac{R_{(d) 0}^{(\tau)} R_{(d) 0}^{(\varepsilon)}+\omega^{2}\left(R_{(d) 1}^{(\varepsilon)}-R_{(d) 0}^{(\tau)} R_{(d) 2}^{(\varepsilon)}\right)}{\left(R_{(d) 0}^{(\varepsilon)}-\omega^{2} R_{(d) 2}^{(\varepsilon)}\right)^{2}+\left(\omega R_{(d) 1}^{(\varepsilon)}\right)^{2}}, \\
B(\omega)=\frac{\omega^{2}\left(R_{(d) 0}^{(\varepsilon)}-R_{(d) 1}^{(\varepsilon)} R_{(d) 0}^{(\tau)}-\omega^{2} R_{(d) 2}^{(\varepsilon)}\right)^{2}}{\left\{R_{(d) 0}^{(\tau)} R_{(d) 0}^{(\varepsilon)}-\omega^{2}\left(R_{(d) 1}^{(\varepsilon)}-R_{(d) 0}^{(\tau)} R_{(d) 2}^{(\varepsilon)}\right)\right\}^{2}} .
\end{gathered}
$$

The complex shear velocity is [14]

$$
v_{c}=\frac{1}{v_{s}}+\frac{k_{2}}{i \omega}=\frac{G_{1}(\omega)+i G_{2}(\omega)}{\varrho},
$$

where $0 \leq s \leq 257, G_{1}(\omega)$ (storage modulus), and $G_{2}(\omega)$ (loss modulus) are, respectively, linked with the nondissipative and dissipative phenomena, and their experimental curves are plotted in Figure 1.

From (47) to (50), the following relations are obtained:

$$
\begin{aligned}
& k_{1}=\frac{\omega \sqrt{\left(\varrho G_{1} / 2\right)\left(\sqrt{1+\left(G_{2} / G_{1}\right)^{2}}+1\right)}}{G_{1} \sqrt{1+\left(G_{2} / G_{1}\right)^{2}}}, \\
& k_{2}=\frac{\omega \sqrt{\left(\varrho G_{1} / 2\right)\left(\sqrt{1+\left(G_{2} / G_{1}\right)^{2}}-1\right)}}{G_{1} \sqrt{1+\left(G_{2} / G_{1}\right)^{2}}} .
\end{aligned}
$$

Let us consider the range of high frequency $\omega_{H} \leq \omega \leq \omega_{U}$ and $\omega \tau \gg 1$ (of order $10^{2}$ ) so that no relaxation phenomena occur (see Figure 1).

By putting

$$
\begin{aligned}
G_{1} & =G_{1 H} 1,001^{s} \\
G_{2} & =G_{2 H} 1,001^{-s},
\end{aligned}
$$




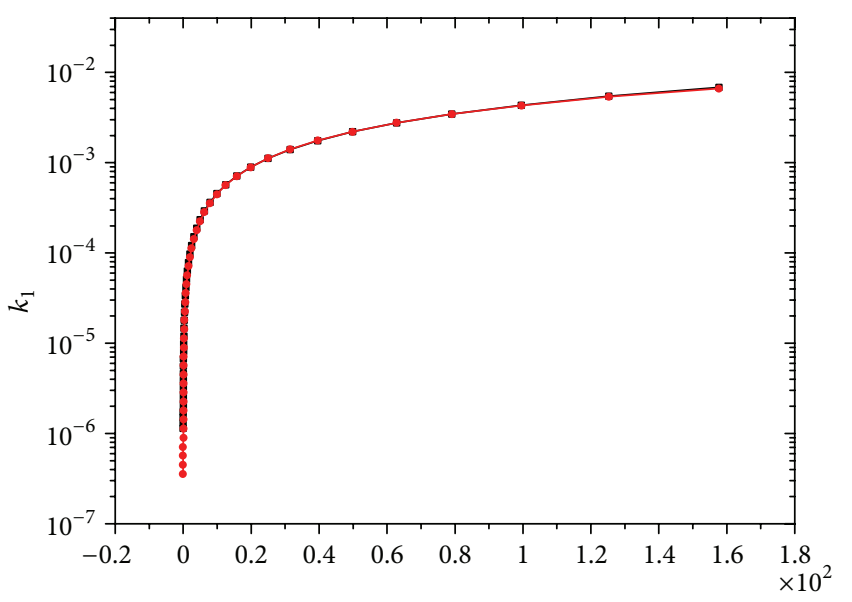
$\longrightarrow k_{1} /\left(\rho_{0} / 2\right)^{0.5}$
$\longrightarrow k_{1} /\left(\rho_{0} / 2\right)^{0.5}$ model

(a)

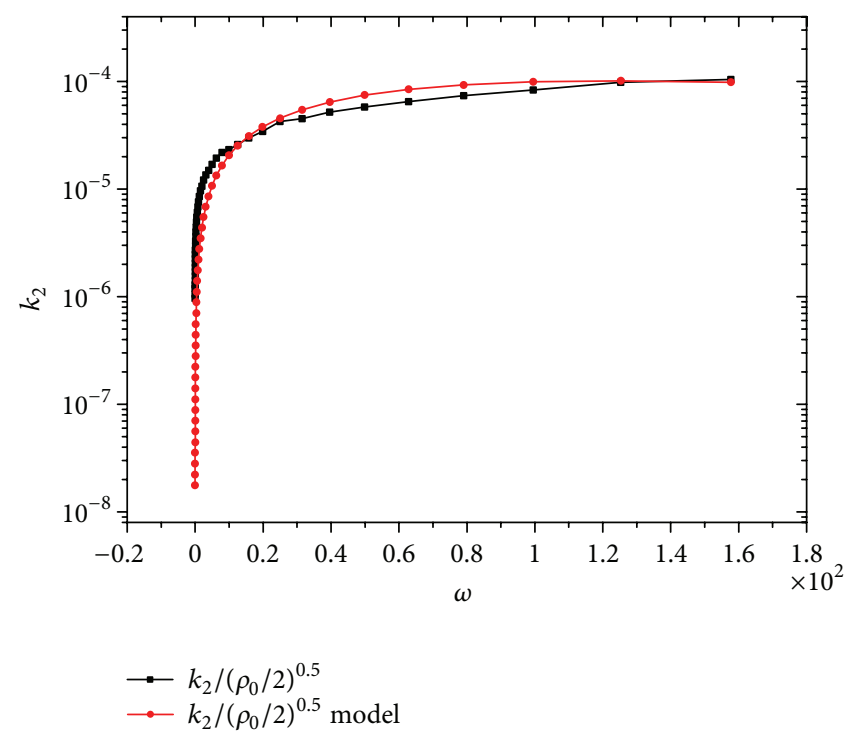

(b)

FIgURE 2: $k_{1}$ and $k_{2}$ for Polyisobutilene: (M.w. $10^{6} \mathrm{~g} / \mathrm{mol} ; T_{0}=273 \mathrm{~K}$ ) the experimental curves (in black) and the theoretical curves (in red) obtained by our model.

where $G_{1 H}=G_{1}\left(\omega_{H}\right)$ and $G_{2 H}=G_{2}\left(\omega_{H}\right),(52)$ becomes

$$
\begin{gathered}
k_{1}=\omega \sqrt{\frac{\varrho}{2}} \sqrt{\frac{\sqrt{\left(G_{1 H}\right)^{2} 1.001^{2 s}+\left(G_{2 H}\right)^{2}}+G_{1 H} 1.001^{s}}{\left(G_{1 H}\right)^{2} 1.001^{2 s}+\left(G_{2 H}\right)^{2}}}, \\
k_{2}=\omega \sqrt{\frac{\varrho}{2}} \sqrt{\frac{\sqrt{\left(G_{1 H}\right)^{2} 1.001^{2 s}+\left(G_{2 H}\right)^{2}}-G_{1 H} 1.001^{s}}{\left(G_{1 H}\right)^{2} 1.001^{2 s}+\left(G_{2 H}\right)^{2}}} .
\end{gathered}
$$

For the Polyisobutilene we have [15] the characteristic values which are

$$
\begin{gathered}
\sigma=10^{-7} \text { sec., } \quad \omega_{H}=3.2 \cdot 10^{14} \mathrm{~Hz}, \\
\omega_{U}=6 \cdot 10^{14} \mathrm{~Hz}, \\
G_{1 U}=2.4 \cdot 10^{9} \mathrm{~Pa}, \quad G_{2 U}=2.75 \cdot 10^{4} \mathrm{~Pa},
\end{gathered}
$$

and the graphics confirm (see Figure 2) with experimental data the validity of the model proposed for viscoanelastic phenomena in continuous media.

\section{Conclusions}

From the viewpoint of nonholonomic irreversible thermodynamics, it is shown that the best specific entropy is an harmonic function in a 27-dimensional manifold. The differential equations of geodetics are obtained and the corresponding curves are explicitly computed. In the linearized theory it is shown that the theoretical results are in agreement with the experimental data in the case of polymeric material (Polyisobutilene) (Figure 2).

\section{Conflict of Interests}

The authors declare that there is no conflicts of interests regarding the publication of this paper.

\section{References}

[1] C. Caratheodory, Undersuchungen über die Grunlagen der Thermodynamik, vol. 2, Gesammelte Mathematische Werke, Munuch, Germany, 1995.

[2] R. Hermann, Geometry, Physics, and Systems, vol. 18, Marcel Dekker, New York, NY, USA, 1973.

[3] R. Mrugała, "Submanifolds in the thermodynamic phase space," Reports on Mathematical Physics, vol. 21, no. 2, pp. 197-203, 1985.

[4] C. Udrişte, O. Dogaru, and I. Ţevy, Extrema with Nonholonomic Constraints: Selected Papers, vol. 4 of Monographs and Textbooks, Geometry Balkan Press, Bucharest, Romania, 2002, Selected papers.

[5] C. Stamin and C. Udrişte, "Nonholonomic geometry of gibbs contact structure," UPB Scientific Bulletin A, vol. 72, no. 1, pp. 153-170, 2010.

[6] J. Merker and M. Krüger, "On a variational principle in thermodynamics," Continuum Mechanics and Thermodynamics, 2012.

[7] G. D. C. Kuiken, Thermodynamics of Irreversible to Diffusion and Rheology, Wiley Tutorial Series in Theoretical Chemistry, John Wiley \& Sons, Chichester, UK, 1994.

[8] G. A. Kluitenberg and V. Ciancio, "On linear dynamical equations of state for isotropic media-I: general formalism," Physica A, vol. 93, no. 1-2, pp. 273-286, 1978.

[9] V. Ciancio and G. A. Kluitenberg, "On linear dynamical equations of state for isotropic media-II. Some cases of special interest," Physica A, vol. 99, no. 3, pp. 592-600, 1979.

[10] V. Ciancio, An Introduction to Thermomechanical of Continuous Media. Rheology, vol. 10 of Monographs and Textbooks, Geometry Balkan Press, Bucharest, Romania, 2009. 
[11] A. Ciancio, "An approximate evaluation of the phenomenological and state coefficients for visco-anelastic media with memory," UPB Scientific Bulletin A, vol. 73, no. 4, pp. 3-14, 2011.

[12] N. G. McCrum, B. F. Read, and G. Williams, Anelastic and Dielectric Effects in Plymeric Solids, John Wiley \& Sons, London, UK, 1967.

[13] P. Hedvig, Dielectric Spectroscopy of Polymers, Adam Hilger, Bristol, UK, 1977.

[14] W. Graham, Comprehensive Polymer Science: the Synthesis, Characterization, Reactions \& Applications of Polymers, vol. 2, chapter 16, edited by G. Allen and J.C. Bevington, Pergamon Press, 1989.

[15] I. M. Ward and D. W. Hadley, Mechanical Properties of Solid Polymers, John Wiley \& Sons, 1993. 


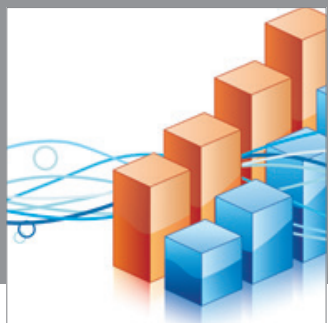

Advances in

Operations Research

mansans

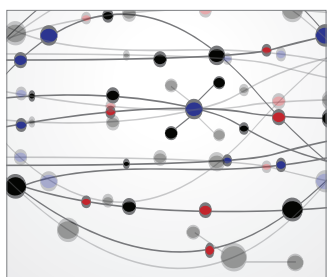

The Scientific World Journal
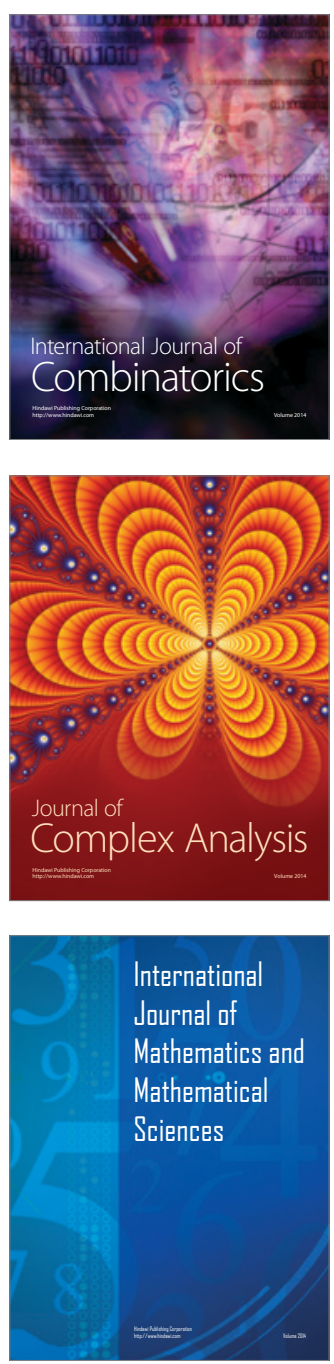
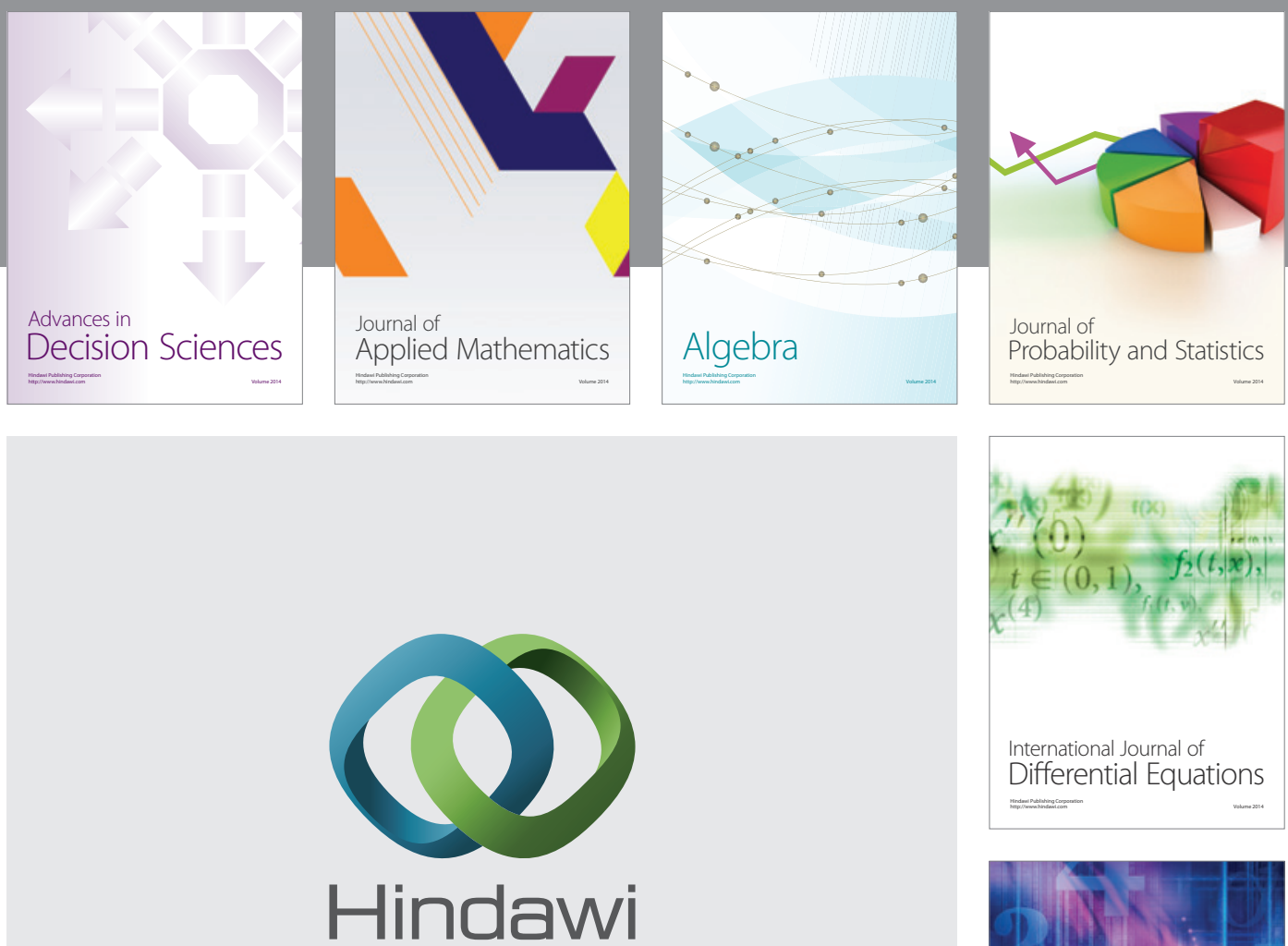

Submit your manuscripts at http://www.hindawi.com
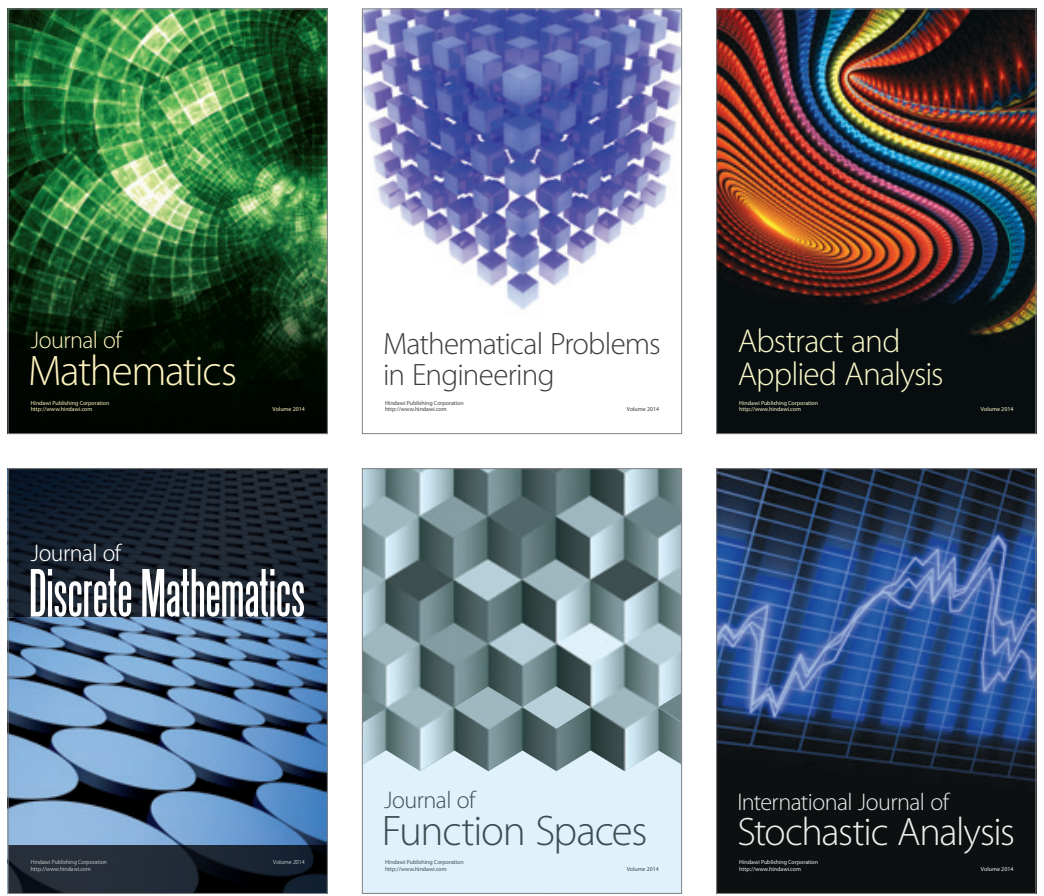

Journal of

Function Spaces

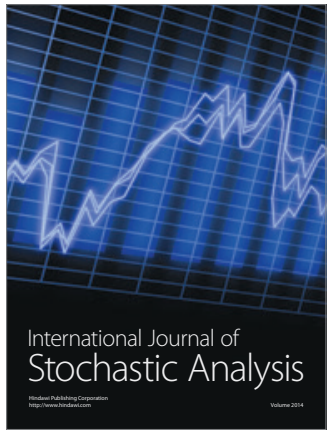

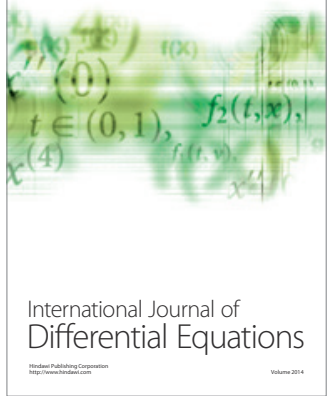
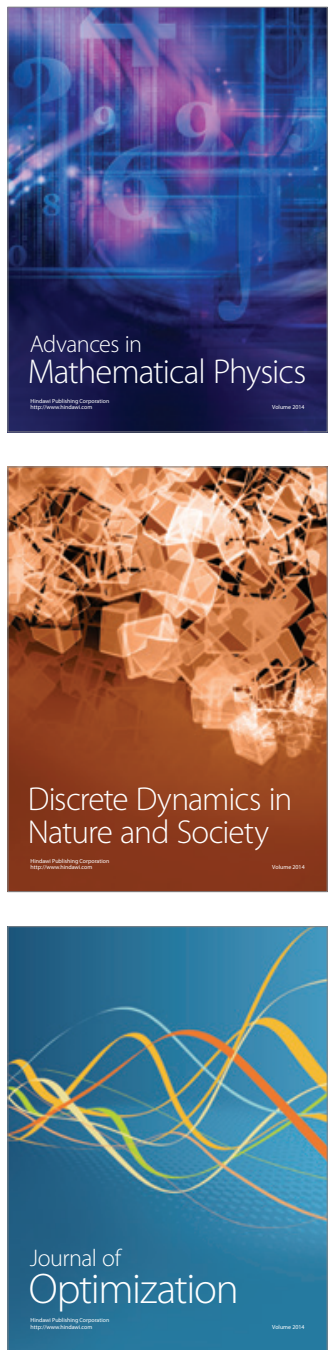